Voix et Images

volxetimages

\title{
Daniel Danis. Imaginer des mondes, réinventer le drame
}

\section{Gilbert David}

Volume 40, numéro 1 (118), automne 2014

Daniel Danis

URI : https://id.erudit.org/iderudit/1028016ar

DOI : https://doi.org/10.7202/1028016ar

Aller au sommaire du numéro

Éditeur(s)

Université du Québec à Montréal

ISSN

0318-9201 (imprimé)

1705-933X (numérique)

Découvrir la revue

Citer cet article

David, G. (2014). Daniel Danis. Imaginer des mondes, réinventer le drame. Voix et Images, 40(1), 9-14. https://doi.org/10.7202/1028016ar d'utilisation que vous pouvez consulter en ligne.

https://apropos.erudit.org/fr/usagers/politique-dutilisation/ 


\title{
DA N I E L DAN IS \\ IM A G INER DES MONDES, RÉINVENTER LE DRAME
}

\author{
$+++$ \\ GILBERT DAVID \\ Université de Montréal
}

\begin{abstract}
À la mémoire de Marie-Aude Hemmerlé, décédée prématurément en juin 2013, afin de souligner l'apport de ses travaux sur le théâtre de Daniel Danis, qui ont laissé une trace durable ${ }^{1}$.
\end{abstract}

La carrière de dramaturge de Daniel Danis (né en 1962) a débuté voilà une vingtaine d'années avec la création de Celle-là (Thêâtre ESPACE GO, Montréal, janvier 1993). Cette production inaugurale, bien servie par une mise en scène inspirée de Louise Laprade et par une solide distribution, n'aura pas connu le succès public escompté... Les critiques ${ }^{2}$ et les gens du milieu théâtral ne se sont pas gênés alors pour déclarer péremptoirement: «Ce n'est pas du théâtre!» En un sens, comme naguère l'emploi péjoratif du terme «impressionniste» pour conspuer la peinture non académique, ce rejet saluait involontairement l'émergence d'une dramaturgie discordante, d'un théâtre-récit polyphonique qui bousculait bon nombre de conventions du drame dialogué, du personnage, de la fable et de l'action ${ }^{3}$.

La dramaturgie de Danis fait irruption à la suite de la génération douée des René-Daniel Dubois, Michel Marc Bouchard et Normand Chaurette, laquelle a fortement marqué les années $1980^{4}$ en s'ouvrant à une pluralité formelle et langagière,

1 Outre sa thèse, Représentations et figurations des dramaturgies québécoises contemporaines. De Normand Chaurette à Daniel Danis (Paris, Université Sorbonne Nouvelle-Paris 3, 2010, 352 f.), Marie-Aude Hemmerlé a publié, à compter du milieu des années 2000, trois importantes études sur le thêâtre de Danis. Voir la «Bibliographie de Daniel Danis» préparée par Camille Robidoux-Daigneault, à la fin du présent dossier, p. $125-146$.

2 À l'exception notable de Robert Lévesque, qui, dans Le Devoir, a d'emblée accueilli l'œuvre de Danis avec un enthousiasme bien senti.

3 Ce qu'il est convenu d'appeler la «mise en crise du drame» ne date pas d'hier, du moins en Europe, où elle s'est manifestée entre 1880 et 1930, avec des auteurs de la modernité comme August Strindberg, Maurice Maeterlinck, Anton Tchekhov, Luigi Pirandello ou Bertolt Brecht. Or, cette crise a persistê jusqu'à nos jours en interrogeant les formes et les principes hérités de la modernité, le tout culminant avec l'avènement du thêâtre postdramatique. Au Québec, c'est avec la dramaturgie exploréenne d'un Claude Gauvreau, puis avec l'œuvre de Michel Tremblay que l'écriture dramatique rompt avec le cadre étroitement mimétique du drame.

4 Voir Jean Cléo Godin et Dominique Lafon, Dramaturgies québécoises des années quatre-vingt: Michel Marc Bouchard, Normand Chaurette, René-Daniel Dubois, Marie Laberge, Montréal, Leméac, coll. «Essai Thêâtre», 1999, 263 p. 
propice à l'éclatement du cadre identitaire mis en place par «le nouveau théâtre québécois ${ }^{5}$ » dans les décennies précédentes. Danis, pour sa part, se démarque à son tour sur la scène québécoise, parallèlement à Carole Fréchette et à Larry Tremblay - ses aînés dont les œuvres gagnent alors en importance -, en adoptant certes un registre parlé, mais tantôt familier, tantôt littérarisé ${ }^{6}$, et en détournant le spectateur de l'identification au premier degré par le recours à l'action narrée.

Un accueil initial plutôt mitigé n'a pas empêché Danis de persister dans sa démarche singulière de poète postmoderne: son œuvre comprend à ce jour plus de vingt titres en thêâtre, dont six textes destinés au jeune public (sans exclusive) et un livret d'opéra (produit en Écosse, dans sa version anglaise, confiée à la traductrice Linda Gaboriau). Avec les années, la dramaturgie de cet auteur n'a eu de cesse d'avoir un grand retentissement au Québec et à l'étranger ${ }^{7}$, tout particulièrement en Europe, ce que confirment l'attribution de nombreux prix, un grand nombre de traductions en plusieurs langues et un ensemble impressionnant de productions théâtrales.

Plusieurs chercheurs n'ont pas tardé à examiner les particularités de cette écriture dans la perspective d'une "dramaturgie de la parole» (notamment Jane $\operatorname{Moss}^{8}$ ), ce qui s'explique aisément par la «surconscience linguistique ${ }^{9}$ » qui l'anime à l'évidence. Parallèlement, d'autres analyses se sont penchées sur la tripartition du «parleur» danisien ${ }^{10}$ ou sur l'hybridation singulière du dialogue et du récit dans sa dramaturgie, alors que plusieurs autres études ont porté sur un seul texte ou quelques-uns en particulier ${ }^{11}$. En dépit de l'abondance relative et de la pertinence des travaux consacrés à l'écriture de Daniel Danis jusqu'à maintenant, il est apparu

5 Voir Michel Bélair, Le nouveau théâtre québécois, Montréal, Leméac, coll. «Dossiers», 1973, 205 p.

6 Voici un exemple représentatif de cette langue d'auteur clairement hybride: «LÉO. Aide-moi, Éva, ma patience s'engoudronne dans une mare de découragement. Un gardien tout nu qui fuckaille derrière une haie; on aura tout vu!» Daniel Danis, Le langue-à-langue des chiens de roche, Montréal, Leméac, 2007, p. 38.

7 Notons ici que l'œuvre de Danis a trouvé peu d'écho dans les pays anglo-saxons, que ce soit au Canada anglais, aux États-Unis ou en Grande-Bretagne, contrairement à une réception favorable en France, en Allemagne, en Italie et dans les pays scandinaves où, il est vrai, le théâtre-récit, sous des déclinaisons diverses, est pratiqué par des auteurs comme Valère Novarina, Olivier Cadiot, Dea Loher, Arne Lygre ou Jon Fosse, pour s'en tenir à cette courte liste.

8 «La dramaturgie de la parole explore à dessein l'emploi de divers registres de langue, afin de créer des persona portant un masque en public, mais aussi d'exprimer émotions et désirs intérieurs.» Jane Moss, «Daniel Danis et la dramaturgie de la parole», Betty Bednarski et Irène Oore (dir.), Nouveaux regards sur le théâtre québécois, Montréal/Halifax, XYZ éditeur/Dalhousie French Studies, coll. «Documents», 1997, p. 117.

9 Voir Lise Gauvin, Langagement. L'écrivain et la langue au Québec, Montréal, Boréal, 2000, 254 p.

10 J'ai proposé, dans un texte publié en 2002, de distinguer chez Danis trois instances - le conteur, le témoin et l'acteur (en tant que voix) — qui se conjuguent dans l'énonciation du parleur. Un tel dispositif exige du comédien une gymnastique verbale très particulière: il lui faut raconter la fable sans pour autant négliger les affects qui accompagnent l'action narrée, tout en se gardant bien de surjouer la situation. Le lecteur voudra bien se reporter à "L'infléchissement parabolique dans Le chant du Dire-Dire de Daniel Danis", l'étude que je cosigne avec Geneviève Brousseau Rivet dans le présent dossier (p. 57-70), pour de plus amples développements.

11 La section «Études» dans la bibliographie de notre dossier répertorie quelque vingt-cinq articles consacrés en tout ou en partie au théâtre de Danis. On y trouvera entre autres abordées des questions reliées à la violence familiale (David Blonde), à la polyphonie du récit dramatisé et à la dilatation du temps (Pauline Bouchet, Nadine Desrochers, Marie-Aude Hemmerlé, Jean-Pierre Ryngaert) ainsi qu'à la poétique mémorielle (Marie-Christine Lesage). 
nécessaire de mener plus avant la réflexion sur ce corpus, au moment où l'auteur, après une période consacrée à la production scénique d'expériences performatives, annonce son intention de revenir à sa "chambre d'écriture ${ }^{12}$ ».

Ainsi, le présent dossier se donne-t-il comme principal objectif de fouiller à nouveaux frais les matériaux de l'imaginaire danisien, sous l'angle de leurs substrats dramatico-narratologiques et anthropo-sociologiques. Le thêâtre de Danis convoque en effet des mondes où s'entremêlent des réalités on ne peut plus triviales et des composantes animistes, voire surnaturelles. Il s'agit maintenant d'en examiner le fonctionnement et d'en extraire les principes actifs. Si tant est que l'on puisse y dissocier la narrativité en tant que telle de son support langagier, il y a lieu de s'interroger sur les stratégies discursives qui déterminent de telles affabulations, diversement anxiogènes, par lesquelles le "vivre-ensemble» est secoué par des conflits entre la communauté et certains de ses membres en rupture de ban ou encore entre des figures d'autorité et des êtres - souvent des enfants - qui se font volontiers héroïques, sans ignorer les conséquences de telles configurations sur la parole et l'action de ses agents. De plus, cette œuvre est hantée - il y a une dimension spectrale dans ce thêâtre onirique - par des inquiétudes à l'égard de la vie - osons le mot - spirituelle de nos contemporains, frappés par les désordres du désir, par les épreuves de la maladie physique ou psychique, et par les violences économiques et idéologiques. Or, tous ces matériaux resteraient inertes s'ils n'étaient pas l'objet d'une intense concaténation poétique. Apparemment, il en va ici d'une politique de l'excès dans le déversement événementiel auquel le spectateur est exposé: on y détecte pourtant des aspérités, des images insolites, des modalités analogiques qui posent autant de défis à l'interprétation - dans tous les sens du terme.

Les sept études réunies dans le présent dossier couvrent un vaste ensemble de questionnements de nature à mettre au jour les divers ancrages socioculturels de la fiction danisienne ainsi que les procédés narratifs mis à contribution dans ce qu'il convient d'appeler la refondation du drame sur d'autres bases que celles qui ont cours dans la tradition mimétique du théâtre en Occident, y compris au Québec. Hervé Guay, dans «Aux origines du thêâtre de Daniel Danis. Mythes de destruction et de création, rites et magie", s'intéresse au caractère syncrétique des mythes que convoque l'auteur dans plusieurs pièces et qui puisent à plusieurs sources: entre autres, la Bible et le christianisme, l'hindouisme, le judaïsme et la spiritualité amérindienne. Au-delà de ce foisonnement, l'analyse discerne dans cette dramaturgie deux grandes structures mythiques, la création et la destruction, dont l'interaction dynamise les relations entre les groupes et entre les personnages. Ces mythes, fondateurs si l'on veut, sont à leur tour inséparables de la ritualisation de la fable où se manifestent quantité de rituels et plusieurs recours à des phénomènes surnaturels.

Les deux études suivantes se penchent sur deux textes majeurs de Danis. Avec l'essai, volontiers spéculatif, sur "L'infléchissement parabolique dans Le chant du Dire-Dire de Daniel Danis», que je cosigne avec Geneviève Brousseau Rivet, il s'est agi de déplier les différentes strates de la narration où s'entremêlent récit de vie et témoignages des trois frères Durant au fil d'une étourdissante suite de péripéties,

12 Voir l'entretien que m'accorde plus loin l'auteur (dans le présent dossier, p. 15-26). 
ce qui conduit à en dégager une double lecture parabolique qui scelle finalement le statut métadramatique de l'œuvre, ouverte dès lors à une interprétation sans fin. En plus de cette dimension autotélique, il faut certainement accorder de l'intérêt à la posture paratopique de l'auteur, qui prend ses distances à l'égard de la naturalisation mimétique du verbe dans le champ théâtral québécois tout en y allant de ses propres images verbales et de ses néologismes, lesquels relèvent d'un procédé d'« étrangéisation", afin de concrétiser sa place comme créateur à part entière.

De son côté, Audrey Camus s'arrête à la romanisation du texte de théâtre avec son étude sur "Le corps du roman dans e. Roman-dit», à partir de la célèbre notion de Bakhtine concernant la contamination des autres genres littéraires par le roman. Ici encore, l'analyse relève la nature composite du matériau narratif, lequel recourt à l'épopée, au conte et à la chanson de geste, qu'elle interprète comme une résurgence contemporaine de la ménippée ou du comique sérieux. S'ensuit alors l'examen de l'hybridation de l'oralité et de la littérarité dans l'écriture, mais aussi des tons - tantôt trivial, tantôt solennel - et des matériaux, dont ceux tirés des rêves ou des arts visuels. Cette esthétique du mélange est l'orientation formelle donnée par Danis à l'histoire d'un peuple métissé dont les errances témoignent justement de la recherche d'un lieu autre pour parler du monde actuel.

Dans «La validation du récit par la poétique de la parole», Jean-Pierre Ryngaert cherche à son tour à cerner en quoi l'accumulation d'épisodes, souvent violents, dans le thêâtre de Danis se trouve à prendre le contrepied des usages dominants des «raconteurs d'histoire» dans l'espace public contemporain. Raconter ne suffit pas, encore faut-il savoir jouer des modalités énonciatives que l'acte de parler libère chez le locuteur danisien. Cette poétique de la parole est certes marquée par le Gestus de la comparution, du témoignage d'après les faits - temporalité de l'aprèscoup et responsabilité de dire le vrai -, mais aussi par la vivacité émotionnelle de la mise en confidence et du commentaire, à même un dispositif apte à faire du spectateur un partenaire du chœur des parleurs. Là est l'originalité de cette choralité à la fois intime et publique, objectivante et parfaitement subjective.

Joseph Danan examine pour sa part, dans «Kiwi ou le vacillement des frontières", quatre façons qu'a Danis de se jouer des catégories habituelles de la dramaturgie jeune public, que ce soit: a) par la porosité entre thêâtre et roman, ce qui débouche sur un traitement singulier de l'image et d'une temporalité aux contours flous; b) par la prise en charge par l'auteur de l'écriture scénique de son texte, ce qui annonce une période d'expérimentation du côté de la performance; c) par un questionnement sur l'espèce de tabou qui concerne les sujets légitimes dans le théâtre jeune public, du fait notable que Danis aborde la sexualité de ses personnages avec franchise et délicatesse; d) par le déplacement d'une dramaturgie danisienne fortement enracinée dans le territoire québécois vers l'exploration de tous les ailleurs, où se manifestent notamment les violences faites aux jeunes.

Marie-Christine Lesage prend en quelque sorte le relais des réflexions conclusives de l'étude précédente avec " "Dans le liquide du récit". Daniel Danis, écrivain scénique». Elle souligne le fait que l'auteur, après avoir composé une série impressionnante de pièces tout au long de la décennie 1993-2003, amorce un virage majeur au milieu des années 2000 en poursuivant un programme exploratoire de 
formes scéniques audacieuses et souvent déroutantes. L'analyse ici porte sur le contexte thêâtral au seuil du nouveau millénaire et sur le devenir scénique d'un auteur dramatique dès lors qu'il prend les commandes du plateau. C'est le statut même du texte qui s'en trouve ainsi relativisé - mais non évacué - alors que $s^{\prime}$ offrent à $l^{\prime}$ «écrivain de plateau ${ }^{13}$ " plusieurs langages, notamment audiovisuels et scénographiques, sans oublier la gestuelle et la profération des acteurs, pour ne pas dire des performeurs. Émerge ainsi un imaginaire radicalement sensoriel, qui renonce à élaborer une fable pleine au profit d'un paysage mental, livré aux associations libres du spectateur.

Dans l'étude qui boucle notre dossier, «Un désir de refondation. Formes de socialité dans l'œuvre dramatique de Daniel Danis», Yves Jubinville entreprend de réfléchir aux tenants et aboutissants du "vivre-ensemble» dans plusieurs œuvres de l'auteur. L'analyse emprunte à la sociologie et à l'anthropologie afin de tester des hypothèses touchant à la référence aux origines ensauvagées du monde danisien sans pour autant y voir un passé érigé en mythe fondateur -, mais renvoyant aussi aux tensions qui affectent ses membres volontiers en rupture de ban dans leurs rapports avec les représentants de l'autorité et de la bien-pensance. Ce faisant, le théâtre de Danis exposerait des mondes livrés à la logique de dissolution du "commun", mais en même temps animés par des mouvements de résistance qui mènent à la recherche d'une communauté réinventée, en mesure de se dresser face à la société administrée. D'où, peut-on penser, la volonté affichée dans cette dramaturgie de privilégier les figures marginales et métissées dans la reconquête, jamais acquise mais toujours espérée, d'une liberté pour tous - visée utopique qui se nourrit de questionnements multiples sur la modernisation rapide de la société canadienne-française, en exhumant les traces de ce que ses agents ont pu refouler...

Nul dossier de revue ne peut prétendre avoir épuisé son sujet... En invitant sept chercheurs à se pencher sur le thêâtre de Daniel Danis, j'ai souhaité rassembler des contributions capables de cerner les principaux enjeux poétiques et socio-anthropologiques d'une écriture dramaturgique qui, à tout le moins, se singularise tant en raison de ses avancées formelles et langagières que de sa résonance avec les préoccupations actuelles de l'humanité. Le temps était venu, m'a-t-il semblé, de donner à lire un ensemble d'études qui risquent des interprétations originales sur les multiples facettes de la recherche dramaturgique et scénique d'un auteur traversé par ce qu'il convient de nommer la "pulsion rhapsodique ${ }^{14}$ ", nourrie de mille et une histoires et de partitions

13 Voir, concernant cette notion qui entend remplacer celle de «mise en scène», la série de six essais de Bruno Tackels, sous la rubrique «Écrivains de plateau», parus aux éditions Les Solitaires Intempestifs (Besançon), de 2005 à 2013.

14 La notion est mise de l'avant et développée par le travail de longue haleine de Jean-Pierre Sarrazac sur l'auteur-rhapsode: voir notamment son essai Théâtres du moi, théâtres du monde, Rouen, Médianes, coll. «Villégiatures/Essais», 1995, 360 p. En quelques mots, le concept de rhapsodie dramaturgique renvoie à l'assemblage kaléidoscopique du dramatique, du lyrique et de l'épique, comme à celui créé par le retournement constant du tragique et du comique. 
parlantes. Il ne me reste plus qu'à souhaiter que ce dossier serve de ferment à des recherches qui sauront en prolonger les résultats. 